\section{Diagnosis Related Group}

Helga Peter ${ }^{1}$ und Thomas Penzel ${ }^{2}$

${ }^{1}$ Marburg, Deutschland

${ }^{2}$ Interdisziplinäres Schlafmedizinisches Zentrum, Charité -

Universitätsmedizin Berlin, Berlin, Deutschland

\section{Definition}

Diagnosebezogene Vergütungsgruppen.

Siehe auch $\triangleright$ „Gesundheitspolitik“.

\title{
Synonyme
}

DRG 